\title{
Routine papillomavirus antigen staining of cervical punch biopsy specimens
}

\author{
D JENKINS, S K TAY, P H MADDOX \\ From the Department of Histopathology, Whittington Hospital, London
}

SUMMARY Immunocytochemical staining for papillomavirus antigen was carried out on 1147 consecutive cervical punch biopsy specimens over 12 months. Of 876 cases with cervical intraepithelial neoplasia (CIN) 351, were antigen positive and of 49 cases with histological evidence of human papillomavirus (HPV) infection but no CIN, 14 were positive. There were 204 cases reported to be normal on routine histological examination and 12 cases reported to show features suggestive but not diagnostic of HPV infection. Of the normal group, $24(12 \%)$ were antigen positive and of the equivocal group, two were positive. In 122 of the normal or equivocal groups cytological examination was repeated at the time of colposcopy, and dyskaryosis was reported in 36 . In only four cases was disease shown by HPV antigen staining when there was no diagnostic histological or cytological abnormality. HPV antigen staining assists in the recognition of the range of histological changes associated with productive HPV infection but is an insensitive test and has only limited value in supplementing histological and cytological examinations as a diagnostic aid in routine colposcopic pathology.

Histological assessment of the presence and severity of epithelial abnormality in colposcopic punch biopsy specimens is essential to the conservative management of premalignant lesions of the uterine cervix. The criteria for the diagnosis and grading of cervical intraepithelial neoplasia (CIN) are well established. ${ }^{1}$ Recent evidence has suggested that genital HPV infection is an important aetiological factor in $\mathrm{CIN}^{2-4}$ The malignant potential of low grade CIN lesions associated with HPV infection and that of cervical lesions of simple HPV infection alone has been shown. ${ }^{56}$ It has been suggested that all patients with histological or cytological evidence of cervical HPV infection should be managed in a similar manner to CIN. ${ }^{6}$ The histological features of HPV infection of the cervix have been described ${ }^{78}$ and the correlation between these features and the presence of HPV 6 or 16 DNA has been reported. ${ }^{9}$ There is, however, wide variation in the reported incidence of koilocytosis, the most widely recognised of these features in CIN. ${ }^{1011}$ Immunocytochemical demonstration of HPV antigen provides a readily available test for HPV infection. We report the results of routine immunoperoxidase staining for HPV antigen using a commercial anti-

Accepted for publication 17 December 1986 serum on all colposcopically directed cervical punch biopsy specimens received in 1985 .

\section{Material and methods}

Routine colposcopically directed cervical punch biopsy specimens were fixed in formol sublimate solution and embedded into paraplast. Each block was cut at three different levels and sections at 4-5 $\mu \mathrm{m}$ thickness were stained with haematoxylin and eosin for conventional histological examination. Sections were stained for HPV antigen by a peroxidaseantialkaline peroxidase immunoperoxidase method. After the wax had been removed the sections were first treated with freshly prepared $0.1 \%$ hydrogen peroxide in methanol solution for 10 minutes to block endogenous peroxidase activity and then processed sequentially through $0.5 \%$ iodine in $70 \%$ alcohol to remove mercuric salts. Sections were then incubated with rabbit antiserum against a genus specific bovine papillomavirus capsid antigen (Dako D580, DAKO Ltd, High Wycombe, England) at a 1/300 dilution overnight. After gentle washing with three changes of Tris buffer solution (TBS, pH 7.6) the sections were incubated with swine antirabbit antiserum (Dako Z196) at a 1/55 dilution for one hour, washed gently 
with three changes of TBS, and incubated with horseradish peroxidase and rabbit antihorseradish peroxidase complex (Dako Z113) at a 1/200 dilution for 45 minutes. Freshly prepared $3,3^{\prime}$-diaminobenzene in $0 \cdot 1 \%$ hydrogen peroxide solution was used to develop the peroxidase reaction. Sections were counterstained with Corazzi's haematoxylin for 40 seconds and screened by conventional light microscopy at a magnification of 100. Positive HPV antigen staining was recorded when brown granular staining of the nuclei within epithelial cells was present. In cases of weak staining the sections were scrutinised at a magnification of 250 and equivocal staining was classified as negative. Sections known to be positive for the antigen were included as positive controls and a section from each case incubated with normal rabbit serum in place of anti-HPV antiserum as a negative control.

The biopsy specimens were classified according to the criteria of Buckley et al ${ }^{1}$ and Dyson et al, ${ }^{7}$ simple HPV infection was diagnosed when, in the absence of CIN, typical koilocytes were present in the superficial layers of the epithelium. These cells were recognised by the presence of a perinuclear vacuole and an irregular hyperchromatic nucleus that was usually either curved or elongated. Additional features of multinucleate or binucleate cells, giant cells, and nuclear enlargement were seen in most cases. Simple HPV infection was distinguished from CIN by the presence of a single basal layer and a normal nucleocytoplasmic ratio in the deeper layers of the epithelium.

Suggestive HPV infection was diagnosed when the epithelium showed no features of CIN but some features described above for HPV infection. In these cases typical koilocytes were not seen but some degree of cytoplasmic vacuolation and some nuclear enlargement were usually present. The pronounced nuclear irregularity and changes of shape that feature in typical koilocytes were absent.

\section{Results}

A total of 1165 biopsy specimens were received in the laboratory during 1985 . Of these, $18(1.5 \%)$ were unsuitable for assessment and were excluded. A positive diagnosis of CIN or simple HPV infection was made in 931 cases $(81 \%)$. Of these, 876 cases showed CIN and 49 showed simple HPV infection. No pathology was found in another 204 cases $(18 \%)$. The remaining 12 cases $(1.0 \%)$ showed minor changes classified as suggestive' of HPV infection. Table 1 shows the overall prevalence of HPV antigen positivity and the prevalence in each diagnostic group.

HPV common antigen staining was seen as a dense brown deposit in nuclei in the more mature superficial layers of the epithelium (fig 1a). True positive staining usually had a fine granular refractile appearance, but the intensity of colour was very variable. In cases showing histological evidence of HPV infection but no evidence of CIN HPV antigen positivity was found in $29 \%$ and in cases with CIN $40 \%$ were positive. The staining was often in cells showing typical koilocytosis, characterised by perinuclear vacuolation with nuclei showing the typical irregular outline, twisted morphology, and granular hyperchromatism, or in superficial squames overlying such an area (fig 1b). Even in this type of lesion antigen positivity was extremely patchy and sometimes only occasional cells were antigen positive. Sometimes nuclei were lost from koilocytotic cells during processing and staining of these detached isolated nuclei could be seen. In CIN 3 antigen positivity was rarely seen and was confined to those very superficial layers of maturing squames when they were present.

Minor nuclear abnormalities and cytoplasmic vacuolation classified as suggestive of HPV infection were sometimes seen adjacent to areas of typical koilocytotic change or CIN, or in sections not showing either of these. In the two cases of 12 in the group showing this pattern of minor abnormality, which were antigen positive, the staining was seen as a granular deposit in the nuclei within vacuolated cells, similar in distribution to that of typical koilocytotic areas.

HPV antigen staining was also seen in superficial nuclei in areas of some sections showing only very slight borderline nuclear changes and no distinct cytoplasmic vacuolation, and which had been classified on routine histological reporting as showing no HPV infection or CIN (figs $2 a$ and $b$ ). HPV antigen staining was found in 24 of 204 cases which had been routinely classified as normal.

Table 2 shows the results of cervical cytology obtained under colposcopic control at the time of biopsy from the negative and "suggestive of HPV infection" groups. Positive HPV antigen staining was found in 15 of 90 cases in which repeat cervical cytology was not available but only in four of 78 cases in which cytology had been repeated and was negative.

Table 1 Prevalence of HPV antigen according to histology

\begin{tabular}{llc}
\hline & \multicolumn{2}{l}{ No of cases (\%) } \\
\cline { 2 - 3 } Histology & $H P V$ negative & HPV positive \\
\hline Normal $(\mathrm{n}=204)$ & 180 & $24(12)$ \\
Suggestive of HPV infection & 10 & $2(17)$ \\
$\quad(\mathrm{n}=12)$ & 35 & $14(29)$ \\
HPV infection $(\mathrm{n}=49)$ & 561 & $315(40)$ \\
CIN ( $=876)$ & 6 & 0 \\
Carcinoma $(\mathrm{n}=6)$ & & \\
\hline
\end{tabular}




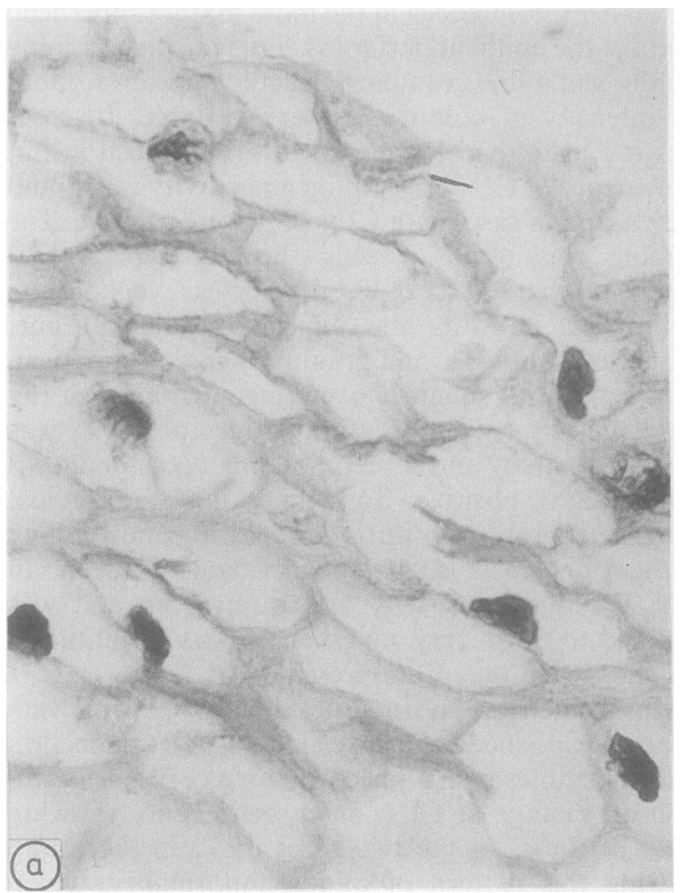

Fig 1 Cervical epithelium from area of HPV infection showing typical koilocytes with irregular nuclei. (a) Nuclei are heavily stained for papillomavirus antigen by immunoperoxidase technique. (b) Haematoxylin and eosin stained section from an adjacent level.

\section{Discussion}

The overall prevalence of HPV antigen positivity and the distribution of antigen staining in this study were similar to that found in previous reports on smaller series. ${ }^{1213}$

A positive diagnosis of CIN or HPV infection was made in 931 cases $(81 \%)$ by conventional histology. In patients with definite histological abnormalities no advantage in routine HPV antigen staining was found. In studies of the prevalence of HPV in CIN or histologically diagnosed HPV infection the numbers of cases with HPV detectable by hybridisation have been much higher than those detected by HPV antigen staining. ${ }^{2}$ In a previous study we showed that the presence of histological evidence of HPV infection closely correlated with HPV detection by DNA-DNA hybridisation using the Southern blot technique on adjacent tissue. ${ }^{9}$ HPV antigen staining therefore seems to be less sensitive in detecting HPV infection than conventional histology when typical histological changes are present. HPV antigen staining detects a

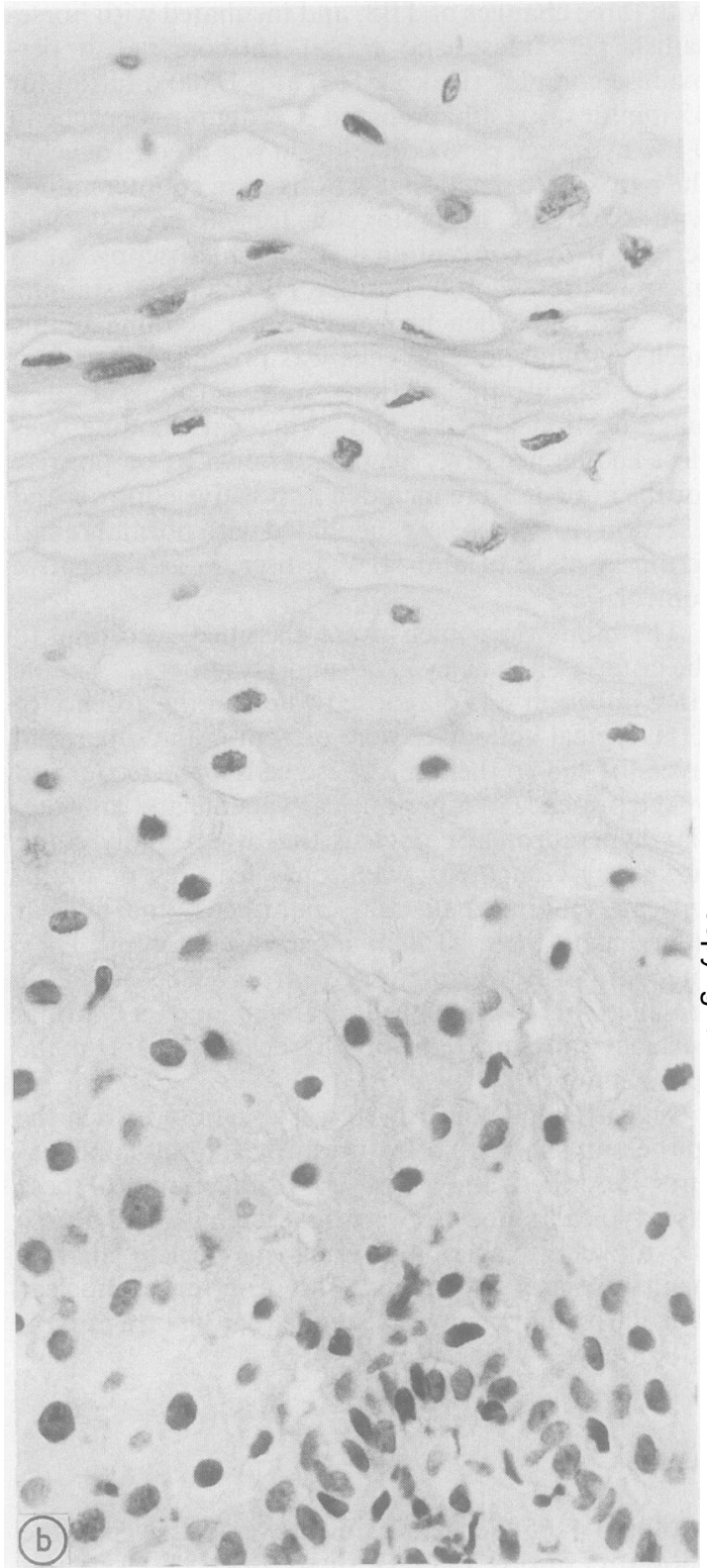

late phase capsid antigen, which only occurs when mature virus particles are being produced. Productive infection only represents one phase of the natural history of HPV infection and also depends on the differentiation of the epithelial cells. This explains the low sensitivity of the investigation as a diagnostic test, although it may have value as a marker of infectivity.

In the remaining 216 cases $(19 \%)$ routine histology had not unequivocally confirmed the colposcopic im- 


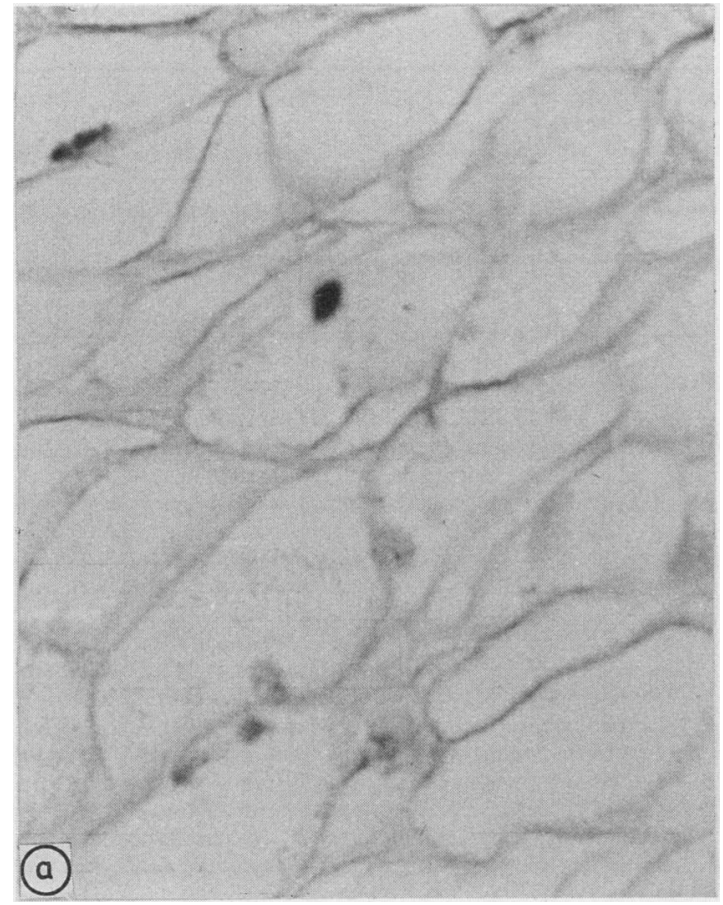

Fig 2 Cervical epithelium showing no distinct histological features of HPV infection or CIN (a) Occasional nuclei in superficial layers are positive for papillomavirus antigen by immunoperoxidase technique (b) Haematoxylin and eosin stained section from adjacent level.

pression of an epithelial abnormality, although some of these cases showed a minor degree of morphological variation from the normal. It is in the diagnosis of these changes that HPV antigen staining could be of most value. Twenty six $(12 \%)$ of these were positive for HPV antigen. The prevalence of antigen staining in the small group showing minor histological abnormalities was not significantly different from that of the larger apparently normal group. These results suggest that HPV antigen staining is of value in confirming HPV infection in patients with suspicious appearances at colposcopy but in whom the biopsy appearances are equivocal or within normal limits. It shows that a considerable range of morphological changes from normality through minor degrees of cytoplasmic vacuolation and nuclear abnormality to typical koilocytosis and CIN can be associated with productive HPV infection. The presence of antigen positivity in $12 \%$ of apparently normal biopsy specimens is comparable with the results of DNA-DNA hybridisation studies on "normal" cervix in which a prevalence of $10-15 \%$ has been reported in most studies, ${ }^{1415}$ although one study reported a

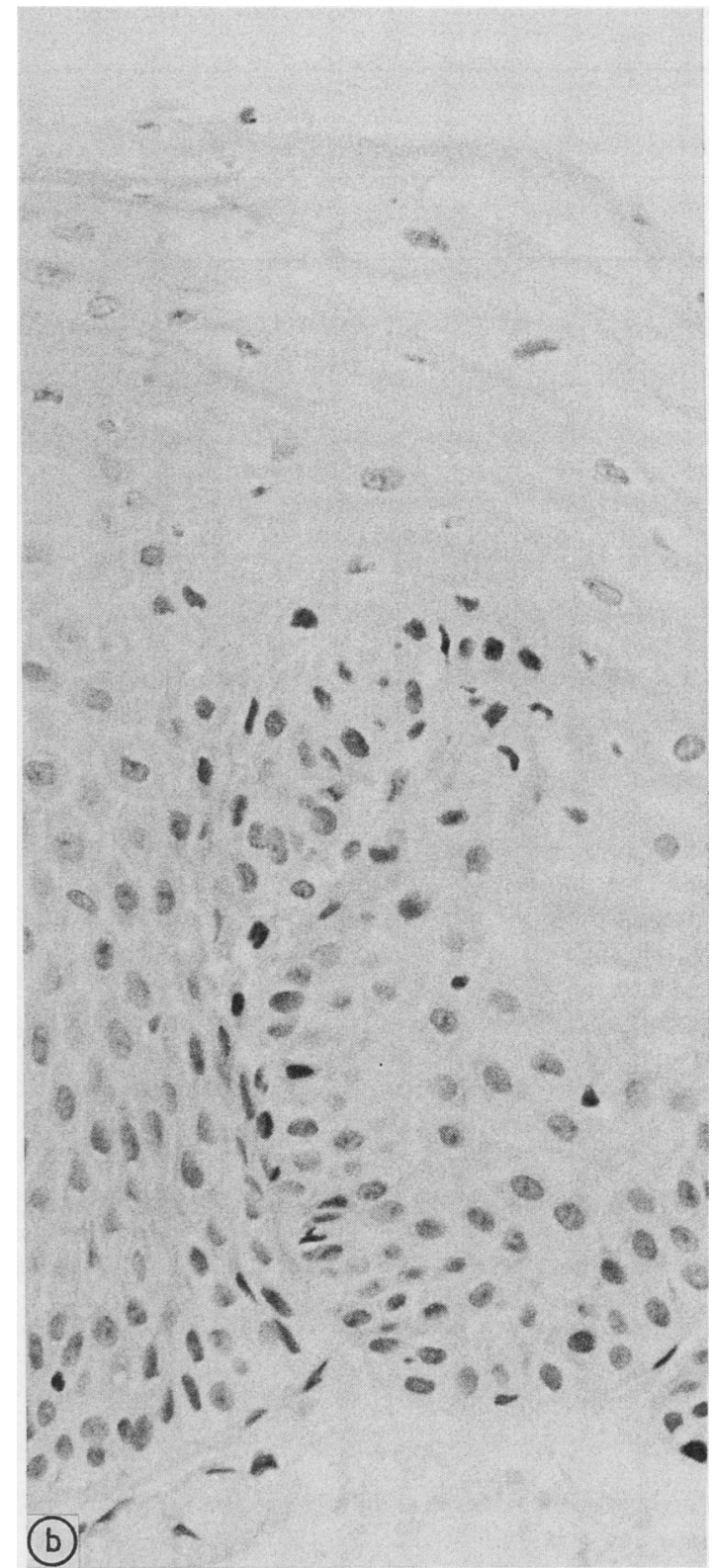

much higher prevalence. ${ }^{16}$ All the patients in our study had previously abnormal cytology, and as with all the other studies, the interpretation is complicated by the difficulty of defining normality. This arises from the use of high risk populations as a source from which "normal" controls are drawn, the use of histologically apparently normal material from patients with CIN elsewhere or possible previous disease, and the variation in histological diagnosis of normality. 
Table 2 Correlation of HPV antigen state and cytological results in 204 cases reported to be normal on routine histological examination

\begin{tabular}{lllllr}
\hline & \multicolumn{5}{l}{ Cytology results of colposcopically directed smears } \\
\cline { 2 - 6 } HPV antigen & Negative & Atypia & Dyskaryosis & Unknown & Total \\
\hline Positive & 4 & 0 & 5 & 15 & $24(12 \%)$ \\
Negative & 72 & 2 & 31 & 75 & $180(88 \%)$ \\
\hline
\end{tabular}

Although the proportion who are antigen positive correspond to the prevalence of HPV DNA detected in other studies of "normal" groups too much importance must not be attached to this, and the value and importance of negative antigen staining in excluding HPV infection must remain uncertain.

When cytology is added to conventional histology HPV antigen positivity was found in only four of 78 cases with normal histology and normal cytology and was not found in any of the eight cases with equivocal histology and normal cytology. this is a pick up rate of only $5 \%$ in the selected group with normal or equivocal histology and normal cytology.

Routine HPV antigen staining on all colposcopic biopsy specimens is of very limited diagnostic value, identifying only four of 1147 cases in which a definitive diagnosis was not made when both histology and cytology had been used. HPV antigen staining has, however, considerable educational value in acquainting the pathologist with the range of appearances associated with HPV infection in the cervix. A positive result can be of diagnostic value in those cases in which no definite histological abnormality is seen in biopsy specimens taken at colposcopy for the investigation of previously abnormal cytology if, however, the practice of performing repeat colposcopically directed cytological examination at the time of biopsy is followed the yield from performing additional HPV antigen staining is very small indeed. HPV antigen staining should usually be confined to those cases in which no definite abnormality can be found either by histological or cytological examinations.

We thank Mr A Singer for permission to study his cases and the staff of the histopathology and gynaecology departments of the Whittington and Royal Northern Hospitals. We also thank the Cancer Research Campaign for generous support to Dr Tay.

\section{References}

1 Buckley CH, Butler GB, Fox H. Cervical intraepithelial neoplasia. J Clin Pathol 1982;35:1-13.

2 McCance DJ, Campion MJ, Clarkson PK, Chester PM, Jenkins $\mathrm{D}$, Singer $\mathrm{A}$. The prevalence of human papilloma virus type 16
DNA sequences in cervical intraepithelial neopolasia and invasive carcinoma of the cervix. Br $J$ Obstet Gynaecol 1985;92:1001-5.

3 Singer A, Walker PG, McCance DJ. Genital wart virus infection: nuisance or potentially lethal? $\mathrm{Br}$ Med $J$ 1984;288:735-7.

4 Zur Hausen H, Gissman L, Schlehoffer JR. Viruses in the aetiology of human genital cancer. Papers in Medicine and Virology 1984;30:170-86.

5 Campion MJ, McCance DJ, Cuzick J, Singer A. progressive potential of mild cervical atypia: prospective cytological, colposcopic and virological study. Lancet 1986;ii:237-41.

6 Evans AS, Monaghan JM. Spontaneous resolution of cervical warty atypia: the relevance of clinical and nuclear DNA features: a prospective study. Br J Obstet Gynaecol 1985;92:165-9.

7 Dyson JL, Walker PG, Dinger A. Human papilloma virus infection of the uterine cervix: histological appearances in 28 cases identified by immunohistochemical techniques. J Clin Pathol 1984;37:126-30.

8 Meisels A, Roy M, Foutier M, et al. Human papilloma virus (HPV) infections of the cervix. The atypical condyloma. Acta Cytol 1981;25:7-16.

9 Jenkins D, Tay SK, McCance DJ, Campion MJ, Clarkson PK Singer A. Histological and immunocytochemical study of CII with associated HPV 6 and 16 infections. J Clin Patho 1986;39:1177-80.

10 Byrne P, Woodman C, Meanwell C, Kelley K, Jordan J. Ko locytes and cervical human papillomavirus infection. Lancet 1986;i:205-6.

11 Jenkins D, Tay SK, Dyson JL. Koilocyte frequency and the prevalence of human papillomavirus infection. Lance 1986;i:557-8.

12 Kurman RJ, Jenson VB, Lancaster WD. Papillomavirus infection of the cervix II. Relationship to intraepithelial neoplasia based on the presence of specific viral structural proteins. Am J Surg Pathol 1983;7:39-52.

13 Ferenczy A, Braun L, Shah KV. Human papillomavirus in condylomatous lesions of the cervix. A comparative ultrastructural and immunohistochemical study. Am J Surg Pathol 1981;5:661-9.

14 Schneider A, Kraus H, Schuhman R, Gissmann L. Papillomavirus infection of the lower genital tract: detection of viral DNA in gynaecological swabs. Int $J$ Cancer 1985;35:443-8.

15 Wickenden C, Steele A, Malcolm ADB, Coleman DV. Screening for wart virus infection in normal and abnormal cervices by DNA hybridisation of cervical scrapes. Lancet 1985; ;:65-7.

16 Cox MF, Meanwell CA, Maitland NJ, Blackledge GB, Scully C, Jordan J. human papillomavirus type 16 homologous DNA in normal human ectocervix. Lancet 1986;:157-8.

Requests for reprints to: Dr D Jenkins, Department of Histopathology, Whittington Hospital, St Mary's Wing, Highgate Hill, London N19 5NF, England. 